\title{
Low-cost Methods for Making 3D Fluidic Polymer and Glass Chips Using Metal Templates
}

\author{
Tomohisa Yamashita, ${ }^{\dagger}$ Kazuyuki Yasukawa, Tomoko Kemmei, Yuuko HoriI, Eriko Nakayama, \\ Tatsuya Muramoto, and Hiroshi TAKADA
}

Toyama Institute of Health, 17-1 Nakataikoyama, Imizu, Toyama 939-0363, Japan

\begin{abstract}
Microfluidics is a rapidly growing field in which small volumes of liquid are moved through channels in a large variety of applications. Fabricating such channels can be expensive. Here, we describe an inexpensive method for making 3D channels in fluidic chips by using a sacrificial template made of coated metal wire or metal tubes. A 3D template is embedded in polymer or glass and then dissolved, leaving channels in the chip, without the need for expensive instruments. By changing the mold, chips of various shapes can be made.
\end{abstract}

Keywords Fluidic chip, 3D chip, PDMS, glass, epoxy, template

(Received April 24, 2015; Accepted July 27, 2015; Published December 10, 2015)

\section{Introduction}

Fluidic chips are used in many fields, ${ }^{1-6}$ such as chemistry and biology. 3D fluidic chips are presently attracting much attention because they can accommodate more channels. Fluidic chips can be made of many materials. Among the many materials for chips, poly dimethylsiloxane (PDMS) and glass are widely used.

PDMS used for chips is usually optically clear and is available commercially (e.g. Sylgard or Silpot). PDMS comes as a highly viscous liquid that turns into a flexible solid by crosslinking polymerization in the presence of a curing agent. Thus, PDMS is well suited for molding complex-shaped chips.

3D fluidic chips are usually made by stacking layers of 2D chips. ${ }^{7}$ However, the stacking method makes it difficult to create arbitrary geometries such as a spiral. Another method for making 3D chips is to use pseudo-3D channels. ${ }^{8}$ Such channels, which are topologically equivalent to planar channels, are generated by bending planar channels in PDMS. Another method is to use a 3D template, such as threads, ${ }^{9}$ wires ${ }^{10,11}$ soldering wires ${ }^{12}$ and soluble organic materials. The templates can be removed by pulling threads or wires out of the PDMS, melting soldering wires or dissolving organic materials. Dissolvable templates include sucrose fibers ${ }^{13}$ and alginate. ${ }^{14}$

Glass is a good material for chips because it is transparent and chemically inert and has been used in many studies. ${ }^{15-18}$ Fabricating a chip, especially a 3D chip, from glass is difficult because glass is brittle and has a high melting point. Common methods for fabricating glass are photolithography and etching, which require expensive instruments or dangerous reagents. 3D glass chips can also be made by direct writing with a femtosecond laser, ${ }^{19,20}$ but it requires an expensive laser system.

$3 \mathrm{D}$ chips can also be made by $3 \mathrm{D}$ printers using various

† To whom correspondence should be addressed. E-mail: tomohisa.yamashita@pref.toyama.lg.jp polymers and metals. Examples of 3D printed chips include propylene fluidic reactionware ${ }^{21}$ and visible transparent resin microchips. ${ }^{22}$ However, the number of materials that can be used in 3D-printing is limited. It is especially difficult to make transparent 3D structures of PDMS or glass with 3D printing.

The high cost of making or buying chips seems to have discouraged some researchers from using chips in their studies. Here, we describe a new and inexpensive method for making 3D channels in PDMS or glass chips using two types of template: coated metal wires and metal tubes.

\section{Experimental}

\section{Reagents and chemicals}

Silpot 184 silicone elastomer was purchased from Dow Corning Toray Co., Ltd. Piano wire (diameter is about $0.3 \mathrm{~mm}$ ), copper tubes, aluminum tubes, aluminum balls and polyvinyl alcohol (PVA) glue were obtained commercially. Polyethylene glycol 2000 (PEG2000), phosphoric acid, hydrochloric acid, sodium hydroxide, ethyl alcohol $(\mathrm{EtOH})$, sodium hypochlorite solution ( $\mathrm{NaClO}$ aqueous solution), alumina (about $45 \mu \mathrm{m}$ ), ceric oxide $\left(\mathrm{CeO}_{2}\right)$, sodium carbonate, light green SFY, naphthol yellow $\mathrm{S}$ and DL-tryptophan were purchased from Wako Pure Chemical Industries (Osaka, Japan). Adenosine 5 -monophosphate, disodium salt (AMP) was obtained from Oriental Yeast Co., Ltd. Glass rod (C1-w) was purchased from Kinari Glass Corp. (Hyogo, Japan). Sheet glass (system 96 SPF100S) was purchased from Spectrum Glass Co., Inc. (USA). Metal casting plaster (G-2) was purchased from Noritake Co., Ltd. (Aichi, Japan). Epoxy resin (super clear resin) was purchased from Fujikyuu Co., Ltd. (Aichi, Japan).

\section{Apparatus}

PDMS and glass were heated with a muffle furnace FO300 or FO310 (Yamato Scientific Co., Ltd., Tokyo, Japan). Liquid flow was controlled with a syringe pump (KD Scientific, USA). 


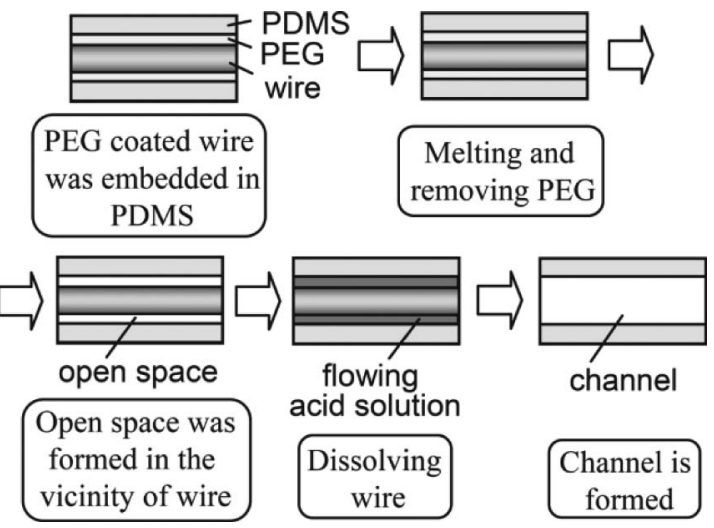

Fig. 1 Schematic view of forming a channel with the coated-wire template method.

As polishing machines, bevel star G15 (Glaster, USA) and MM100 (Proxxon) were used.

\section{Chromatographic measurement}

In chromatographic measurements, Model G1311A HPLC pump, Model 1313A autosampler, and Model G1315B UV detector (Agilent Technologies) were used. Cooling mediums were used in order to prevent from appearing bubbles in HPLC line. Cooling mediums were frozen in a freezer (from -20 to $-30^{\circ} \mathrm{C}$ ) before using for measurements. A flow-rate of $0.3 \mathrm{~mL} / \mathrm{min}$ was used with the following gradient: $0 \mathrm{~min}, 100 \%$ water; $3 \mathrm{~min}, 100 \% 4 \times 10^{-4} \mathrm{M}$ sodium carbonate aqueous solution (linear gradient). The injection volume was $20 \mu \mathrm{L}$, and concentrations of DL-tryptophan and AMP were $8 \times 10^{-6} \mathrm{M}$ and $8 \times 10^{-5} \mathrm{M}$, respectively. The mobile phase was degassed by helium during measurements. The detection wavelength was $280 \mathrm{~nm}$.

\section{Procedures}

Coated-wire template method. In this method, polymer (PEG2000), which melts by heat, was coated on the piano wire. This method was applied to PDMS. Figure 1 shows a schematic illustration of the polymer coating and wire dissolving process. After curing the PDMS, PEG was melted and removed in order to make a space between the wire and the PDMS wall. This space is used for the passage of aqueous acid solution. The piano wire can be removed by dissolution using an aqueous acid solution after curing the PDMS.

Figure 2 shows how a chip is made by the coated-wire template method. A 3D channel template was made with a piano wire (Fig. 2a). The junction was fixed by PVA glue. The PEG was melted at $80^{\circ} \mathrm{C}$ on a hot plate and coated on the piano wire with a brush. The piano wire was allowed to cool at room temperature until the PEG solidified (Fig. 2b). A PDMS base of Silpot 184 and a curing agent were mixed in a 10:1 ratio and degassed. Initially, a little PDMS prepolymer was poured in a container. The container was heated in an $80^{\circ} \mathrm{C}$ oven for $30 \mathrm{~min}$ to cure the PDMS (Fig. 2c). The 3D channel template was placed on the PDMS layer (Fig. 2d), and PDMS prepolymer was added until it covered the template (Fig. 2e). After curing the PDMS at room temperature, excess piano wire outside the PDMS was removed. PEG was melted at $80^{\circ} \mathrm{C}$ on a hot plate and poured into the outlet of the adaptors in order to seal the space between the wire and the interior of the adaptor. And then the adaptors were attached to the piano wires before the PEG solidified (Fig. 2f). A PDMS base and curing agent were mixed

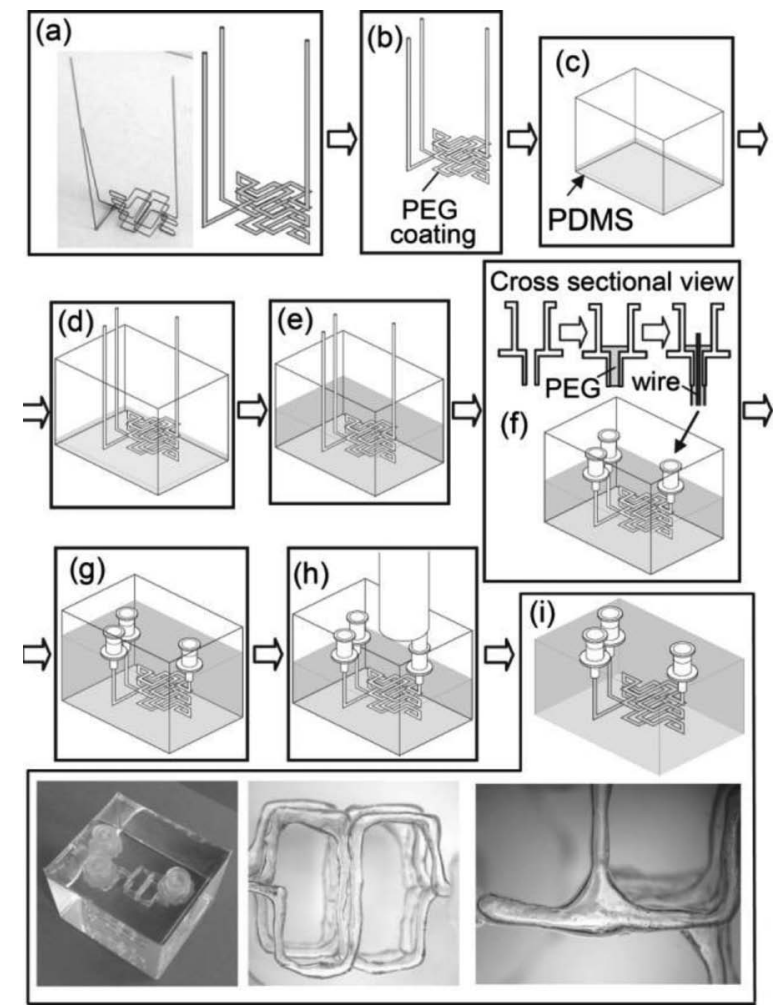

Fig. 2 Schematic illustration of chip making by the coated-wire template method: (a) template was made by piano wire, (b) PEG was coated on wire, (c) PDMS bottom layer was formed in container, (d) template was put on the PDMS bottom layer, (e) PDMS prepolymer was added and cured, (f) surplus wires outside the PDMS were cut, and PEG was filled in outlet of adaptor. This adaptor was put through with the wire, $(\mathrm{g})$ a curing agent and PDMS base were mixed in a 2:10 ratio, and this PDMS prepolymer was added in the container until about half height of adaptor was immersed. PDMS was cured. (h) Chip was warmed in water bath in order to melt PEG. Liquid PEG was pushed out by syringe. (i) Piano wire was removed by phosphoric acid. PDMS thin layer was coated on the surface of the channel, and the chip was taken off from the container.

in a 10:2 ratio. This PDMS prepolymer was degassed and poured on the solid PDMS in the container until it covered half the height of the adaptors (Fig. 2g). After curing the PDMS at room temperature, the container with PDMS was immersed in a hot water bath at $60^{\circ} \mathrm{C}$ to melt the PEG. Liquid PEG was pushed out with a syringe, and the channel was washed with hot water of $60^{\circ} \mathrm{C}$ (Fig. 2h). After removal of the PEG, $40 \%$ phosphoric acid aqueous solution was pushed through the PDMS channel with a syringe pump at $0.05 \mathrm{~mL} / \mathrm{min}$ to dissolve the piano wire (total volume was about $80 \mathrm{~mL}$ ). After the piano wire was dissolved, the channel was washed with water with a syringe pump at $0.1 \mathrm{~mL} / \mathrm{min}$ (total volume was about $20 \mathrm{~mL}$ ). The channel was washed with EtOH by hand, and air was allowed to flow in the channel by air pump in order to dry the channel. After washing with diluted $\mathrm{NaClO}$ aqueous solution, water and $\mathrm{EtOH}$ was injected in order by hand, and air was directed into the channel by air pump in order to dry the channel. The PDMS channel wall was smoothed by applying a coating. PDMS prepolymer and heptane were mixed in a 1:1 ratio. The mixture was then injected into the channel to coat it with PDMS. Excess PDMS prepolymer was removed by flushing the channel with air until the PDMS was completely polymerized. After polymerization of PDMS, the chip was removed from the 
PDMS, glass, etc.
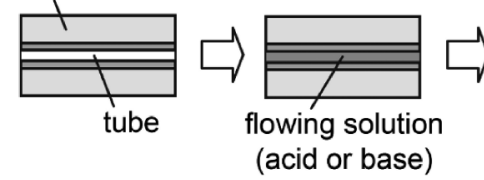

Tube was embedded in PDMS, glass, etc.
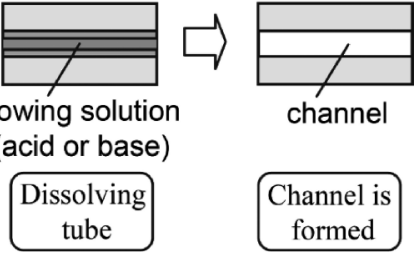

formed

Fig. 3 Schematic view of forming a channel by the tube template method.
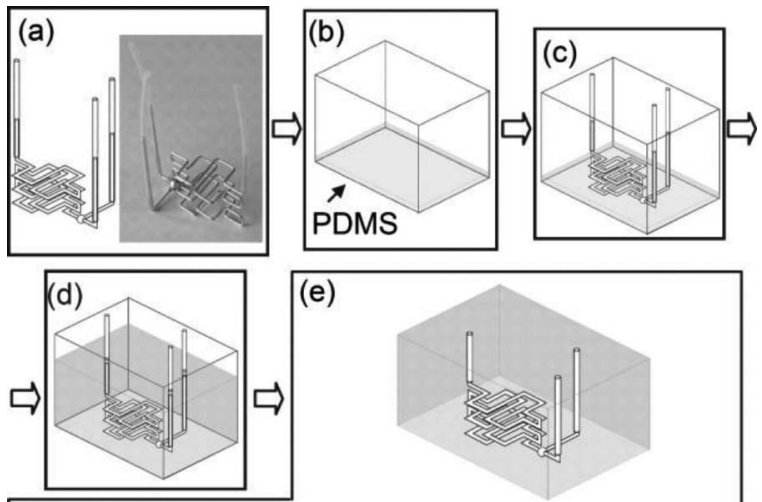

(e)
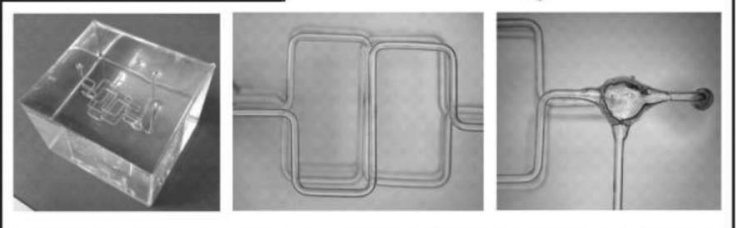

Fig. 4 Schematic illustration of making PDMS chip by tube template method: (a) template was made by aluminum tube, and polyethylene tubes were connected, (b) PDMS bottom layer was formed in container (c) template was put on the PDMS bottom layer, (d) PDMS prepolymer was added and cured, (e) after curing PDMS, aluminum tube was dissolved. After channel was washed, chip was taken off from the container.

container (Fig. 2i). Photos of the procedure are shown in Fig. S1 (Supporting Information)

\section{Tube template method}

Metal tubes such as aluminum and copper were used as the template for making channels with PDMS, glass, etc., as shown in Fig. 3.

\section{PDMS chip}

An aluminum tube (inside diameter $0.2 \mathrm{~mm}$, outside diameter $0.6 \mathrm{~mm}$ ) and an aluminum ball (diameter $3 \mathrm{~mm}$ ) were used as a template for the $3 \mathrm{D}$ channel. Making a T-junction procedure consisted of the following steps: (i) making holes in an aluminum ball by drill, (ii) formation of template with aluminum tubes and (iii) combining with aluminum tubes and ball, and fixing by PVA glue. Templates of aluminum tubes and the ball were washed with EtOH before being combined. Polyethylene tubes were connected to aluminum tubes and fixed by PVA glue (Fig. 4a). A PDMS base of Silpot 184 and a curing agent were mixed in a 10:1 ratio and degassed. Initially, a little PDMS prepolymer was poured in a container. The container was placed in an $80^{\circ} \mathrm{C}$ muffle furnace for 30 min to cure the PDMS
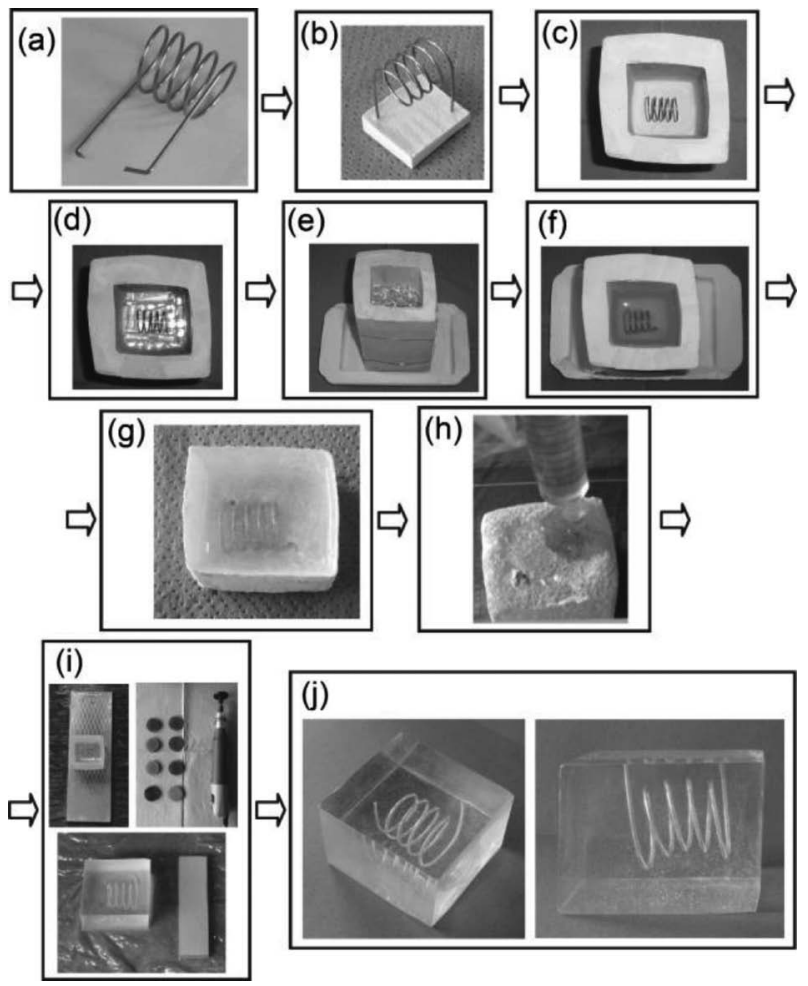

Fig. 5 Schematic illustration of making glass chip by the tube template method: (a) making a template with a copper tube, (b) Placing the template on the plaster, (c) placing the template in a plaster mold, (d) placing cut glass rods in the plaster mold, (e) the plaster mold before heating, (f) the plaster mold after heating, (g) removal of plaster, (h) dissolving the copper tube, (i) polishing glass with various diamond files, ( $j$ ) Views of the glass chip after polishing with a slurry of water and $\mathrm{CeO}_{2}$ and washing.

(Fig. 4b). The template was placed on the PDMS layer (Fig. 4c), and PDMS prepolymer was added until it covered the template (Fig. 4d). This new PDMS was cured in a $60^{\circ} \mathrm{C}$ muffle furnace for 60 min. After curing the PDMS, the PDMS was immersed in a hot water bath at $60-80^{\circ} \mathrm{C}$. Then an aqueous solution of $6 \mathrm{~mol} / \mathrm{L} \mathrm{NaOH}$ was injected into the aluminum tube by hand to dissolve the template. After removal of the template, the channel was washed with flowing water (room temperature), hot water $\left(60-90^{\circ} \mathrm{C}\right)$ and diluted $\mathrm{NaClO}$ aqueous solution in order repeatedly to dissolve residual PVA glue. The channnel was washed with $1 \mathrm{~mol} / \mathrm{L} \mathrm{HCl}$ aqueous solution, hot water $\left(60-90^{\circ} \mathrm{C}\right)$, water (room temperature) and $\mathrm{EtOH}$. The channel was dried by flushing it with air by air pump. The chip was removed from the container, and the channel was flushed with air again. Views of the completed PDMS chip are shown in Fig. 4e. (Polyethylene tubes were already removed) Photos of the procedure are shown in Fig. S1 (Supporting Information).

\section{Glass chip}

A slurry made from mixing 100 parts metal casting plaster with about 45 parts water was poured in a container and allowed to solidify. The plaster mold was removed from the container and dried at room temperature. A 3D channel template was made with a copper tube (inside diameter $0.6 \mathrm{~mm}$, outside diameter $1.0 \mathrm{~mm}$ ), (Fig. 5a) and placed on the plaster (Fig. 5b). The template was placed in the plaster mold (Fig. 5c). Cut glass rods were put in the plaster mold (Fig. 5d, e). The mold was placed in the muffle furnace and heated to melt the glass rods 

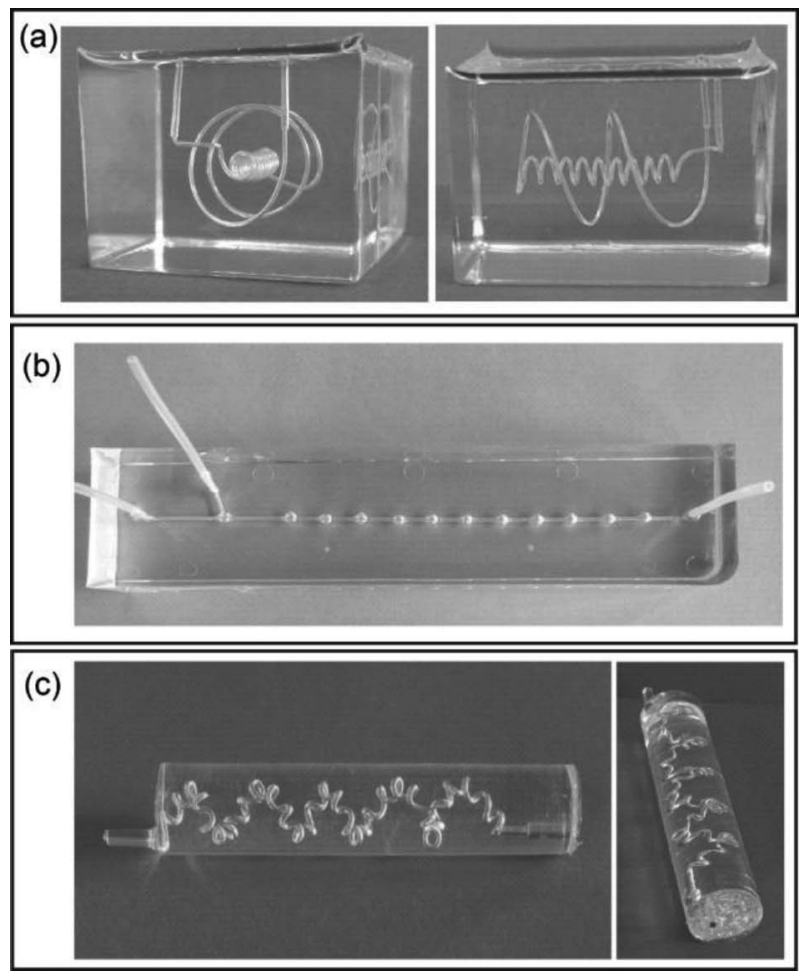

Fig. 6 Chips made by tube template method: (a) epoxy chip: aluminum tube (i.d. $0.6 \mathrm{~mm}$, o.d. $1.0 \mathrm{~mm}$ ) was used for making the template, (b) epoxy chip: aluminum tube (i.d. $0.6 \mathrm{~mm}$, o.d. $1.0 \mathrm{~mm}$ ) and aluminum ball (diameter $3.0 \mathrm{~mm}$ ) was used for making the template, (c) columnar PDMS chip with a channel that was made with an aluminum tube (i.d. $0.6 \mathrm{~mm}$, o.d. $1.0 \mathrm{~mm}$ ) as template.

(time schedule of heating is shown in Fig. S2a (Supporting Information)). After cooling the glass (Fig. 5f), the plaster was removed (Fig. $5 \mathrm{~g}$ ). $\mathrm{HNO}_{3}$ was injected into the copper tube to dissolve the copper (Fig. 5h). After removal of the template, the channel in the glass was washed. The surface of the glass was coarsely polished with diamond files (Fig. 5i), finely polished with a slurry of water and $\mathrm{CeO}_{2}$, and the chip was washed. The finished glass chip is shown in Fig. 5j.

\section{Results and Discussion}

\section{Coated-wire template method}

Piano wire was selected as a template for the channel because it is hard, which makes it well-suited for retaining a 3D structure. Acid solutions such as phosphoric acid and hydrochloric acid can dissolve the piano wire because it is mainly made of iron. Here, we used phosphoric acid because it was found to dissolve iron faster than hydrochloric acid in a preliminary experiment. The advantages of this method are that it is inexpensive and simple. Piano wire is inexpensive. A template of channels can be easily constructed by bending piano wires. A junction can be also made by connecting wires. In Fig. 2a, a T-junction was fixed by water-soluble PVA glue.

Coating materials should have a low melting point and be insoluble in PDMS. For this study, we selected PEG (2000), which has an average molecular weight of 2000. PEG (2000) is soluble in water and insoluble in PDMS. In addition, PEG (2000)'s melting point is less than $100^{\circ} \mathrm{C}$. These properties make PEG (2000) well-suited for a coating material. After
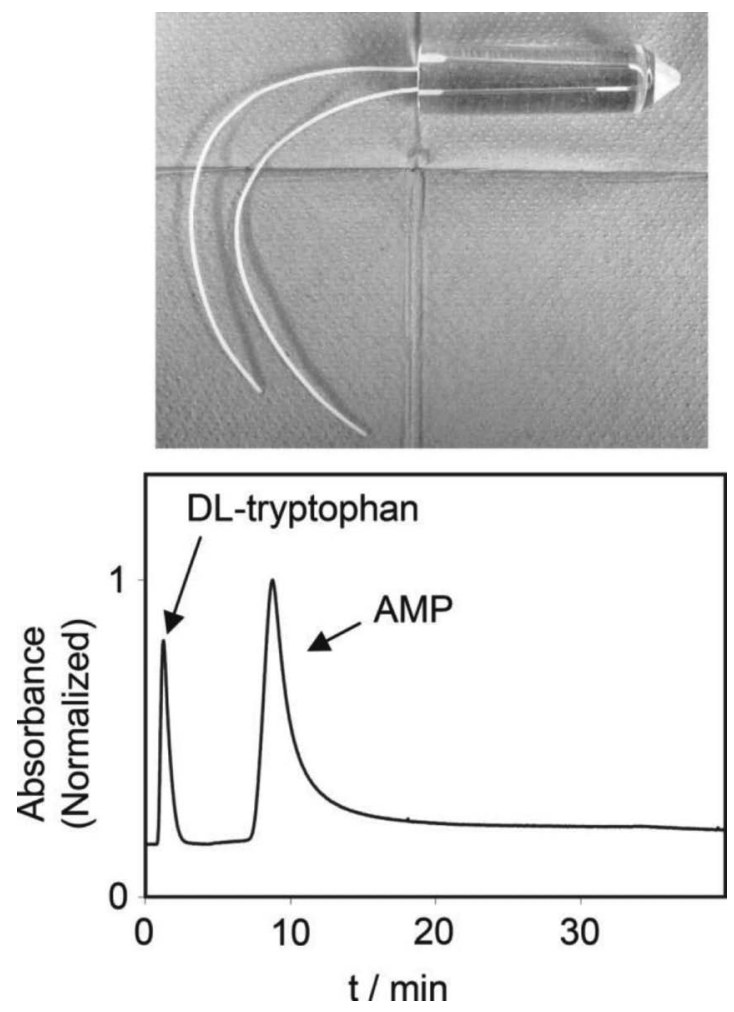

Fig. 7 Chromatography chip and chromatogram of mixture of DL-tryptophan and AMP.

curing the PDMS, the chip was heated to melt the PEG. Liquid PEG can be easily pushed out of a channel by pressure. PEG residue in the channel can be washed away with flowing water. Removal of the PEG creates a space between the wire and PDMS. Aqueous acid solution is injected into this space to dissolve the wire. PEG (20000) can also be used as the coating material, although it is more viscous than PEG (2000) and has a higher melting point, which makes it more difficult to remove from the channel.

Presently, it is difficult to coat a wire evenly with PEG, which causes the channel to have an irregular surface. If a fine coating method is found, it will improve the shape of the channel.

\section{Tube template method}

In this study, the tube template method was explained by making PDMS and glass chips.

\section{PDMS chip}

Aluminum was selected as the tube material because it can be dissolved by both acid and base solutions. We used a basic solution (aqueous $\mathrm{NaOH}$ ) because acids such as hydrochloric acid acting on aluminum sometimes causes the formation of precipitates that clog the tubes, especially narrow tubes. Clogging of aluminum tubes was less frequent when a base such as $\mathrm{NaOH}$ was used. But, in the case of $\mathrm{NaOH}$, the rate of dissolution is very low at room temperature, so the $\mathrm{NaOH}$ solution and chip were warmed before injection to accelerate the dissolution. Once injection of $\mathrm{NaOH}$ solution is started, it should not be stopped. We found that if it is stopped, the tube occasionally became clogged, apparently by precipitates.

In Fig. 4a, a T-junction was structured by aluminum tubes and a ball and fixed by water-soluble PVA glue.

Other chip materials can be used with this method. 
(a)

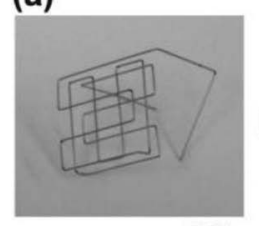

(c)
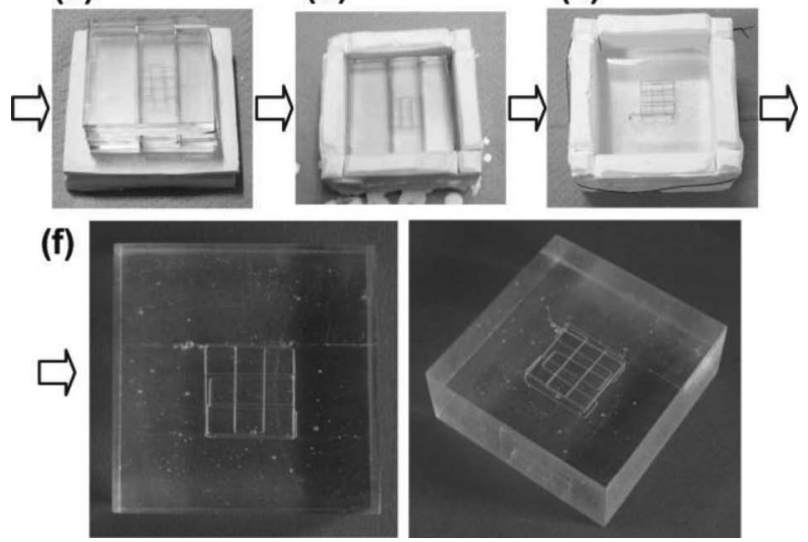

Fig. 8 Procedure of making 3D fluidic glass chip from sheet glass: (a) copper tube template (o.d. $0.3 \mathrm{~mm}$ ), (b) a template was placed on a plaster, (c) sheet glass were set, (d) a template and glass were enclosed with plaster, (e) after heating in an electric furnace, (f) after dissolving template by $\mathrm{HNO}_{3}$ and $\mathrm{HCl}$, glass was polished. The surface of the glass was polished with a polishing machine.

For example, epoxy chips are shown in Figs. $6 \mathrm{a}$ and $6 \mathrm{~b}$. In the case of the epoxy chips, an aluminum tube and aluminum balls were also used as template, and the template was dissolved by aqueous $\mathrm{NaOH}$ solution. Chips of various shapes can be made too. For example, a PDMS chip with a columnar shape is shown in Fig. 6c.

It is possible that these chips can be used for various analytical experiments. For example, in Fig. 7, chromatography chip and chromatograph are shown. Alumina and glass fiber were set in the channel of an epoxy chip, and DL-tryptophan and AMP were separated. Schematic illustration of the HPLC system and photos of the chip making procedure are shown in Fig. S3 (Supporting Information).

\section{Glass chip}

The method for making glass chip in this study is based on kiln work, which is a technique using an electric furnace (kiln). In general, the technique of kiln work has been applied for crafts of glass, metal clay and so on. The electric furnace is used for melting and fusing glasses. ${ }^{23}$ The advantage of kiln work is its inexpensiveness because glass can be transformed without expensive apparatus. Here, a copper tube was selected for the template because (i) its melting point $\left(1083^{\circ} \mathrm{C}\right)$ is higher than that of glass $\left(700-900^{\circ} \mathrm{C}\right)$ (aluminum melts at $660^{\circ} \mathrm{C}$ ), (ii) it is commercially available as electrodes for electrical discharge machining and is inexpensive, while long, thin tubes of other metals are difficult to obtain, and (iii) it dissolves in nitric acid.

Molten glass must be cooled slowly to prevent strain, which can cause cracking of the glass. After annealing, the chip is removed from the furnace, separated from the plaster mold and polished. Polishing is done first with diamond files of different coarsenesses and finally with a slurry of water and $\mathrm{CeO}_{2}$.

Sheet glass also can be used for making fluidic chips. The procedure of making 3D fluidic glass chips from sheet glass is shown in Fig. 8. The time schedule of heating is shown in Fig. S2b (Supporting Information). This method is a little easier than the method described in Fig. 5. However, the structure of chip is limited. In this method, the chip tends to contain bubbles in the glass. Optical microscopic images of a chip made from sheet glass are shown in Fig. S5 (Supporting Information).

\section{Conclusions}

Here, we described low-cost and simple methods for making 3D channels in polymer and glass chips using sacrificial metal templates. By changing the mold, chips of various shapes can be made.

\section{Acknowledgements}

The authors thank Hideki Matsui, Yukio Demachi, Isao Kamide and Naohito Saito for supporting research.

\section{Supporting Information}

Photos of making a PDMS chip by coated-wire template method and tube template method are shown Fig. S1. The time schedule of heating glass rod and sheet glass in an electric furnace is shown in Fig. S2. Schematic illustration of the HPLC system and photos of making procedure are shown in Fig. S3. Chromatograms of DL-tryptophan, AMP are shown in Fig. S4. Optical microscopic images of a chip made from sheet glass are shown in Fig. S5. Photos of mixing of colored water in PDMS chip are shown in Fig. S6. Additional information on chips are shown in Fig. S7. This material is available free of charge on the Web at http://www.jsac.or.jp/analsci/.

\section{References}

1. H. Becker and C. Gartner, Anal. Bioanal. Chem., 2008, 390, 89.

2. D. Schäpper, M. N. H. Z. Alam, N. Szita, A. E. Lantz, and K. V. Gernaey, Anal. Bioanal. Chem., 2009, 395, 679.

3. K. Faure, Electrophoresis, 2010, 31, 2499.

4. F. Xu and Y. Baba, Electrophoresis, 2004, 25, 2332.

5. L. Zhang, F. Dang, and Y. Baba, J. Pharm. Biomed. Anal., 2002, 30, 1645.

6. N. Kitamura, K. Ueno, and H.-B. Kim, Anal. Sci., 2008, 24, 701.

7. M. Zhang, J. Wu, L. Wang, K. Xiao, and W. Wen, Lab Chip, 2010, 10, 1199.

8. H. Wu, T. W. Odom, D. T. Chiu, and G. M. Whitesides, J. Am. Chem. Soc., 2003, 125, 554

9. M. K. S. Verma, A. Majumder, and A. Ghatak, Langmuir, 2006, 22, 10291.

10. Y. F. Jia, J. H. Jiang, X. D. Ma, Y. Li, H. M. Huang, K. B. Cai, S. X. Cai, and Y. P. Wu, Chin. Sci. Bull., 2008, 53, 3928.

11. S. Agrawal, A. Morarka, K. M. Paknikar, and D. Bodas, Microelectron. Eng., 2012, 90, 104.

12. S.-H. Song, C.-K. Lee, T.-J. Kim, I.-c. Shin, S.-C. Jun, and H.-I. Jung, Microfluid. Nanofluid., 2010, 9, 533.

13. J. Lee, J. Peak, and J. Kim, Lab Chip, 2012, 12, 2368.

14. X.-Y. Wang, Z.-H. Jin, B.-W. Gan, S.-W. Lv, M. Xie, and W.-H. Huang, Lab Chip, 2014, 14, 2709. 
15. K. Sato, A. Hibara, M. Tokeshi, H. Hisamoto, and T. Kitamori, Anal. Sci., 2003, 19, 15.

16. N. A. Lacher, K. E. Garrison, R. S. Martin, and S. M. Lunte, Electrophoresis, 2001, 22, 2526.

17. M. Pumera, Talanta, 2005, 66, 1048.

18. A. Smirnova, K. Shimura, A. Hibara, M. A. Proskurnin, and T. Kitamori, Anal. Sci., 2007, 23, 103.

19. K. Sugioka, Y. Hanada, and K. Midorikawa, Appl. Surf. Sci., 2007, 253, 6595 .
20. Y. Liao, J. Song, E. Li, Y. Luo, Y. Shen, D. Chen, Y. Cheng, Z. Xu, K. Sugioka, and K. Midorioka, Lab Chip, 2012, 12 , 746.

21. P. J. Kitson, M. H. Rosnes, V. Sans, V. Dragone, and L. Cronin, Lab Chip, 2012, 12, 3267.

22. A. I. Shallan, P. Smejkal, M. Corban, R. M. Guijt, and M. C. Breadmore, Anal. Chem., 2014, 86, 3124.

23. M. Okuno, "Kiln work-Handmade Glass Using Electric Kiln in Studio", 2008, Holp Shuppan, Publishers, Tokyo. 